\title{
A novel Veress needle mechanism that reduces overshooting after puncturing the abdominal wall
}

\author{
Roelf R. Postema ${ }^{1,2}$ (D) David Cefai ${ }^{3} \cdot$ Bart van Straten $^{1} \cdot$ Rein Miedema ${ }^{1} \cdot$ Latifa Lesmana Hardjo $^{1}$. \\ Jenny Dankelman ${ }^{1} \cdot$ Felix Nickel $^{4} \cdot$ Tim Horeman-Franse $^{1}$
}

Received: 10 February 2021 / Accepted: 6 June 2021 / Published online: 22 June 2021

(c) The Author(s) 2021

\begin{abstract}
Background Complications that occur in laparoscopic surgery are often associated with the initial entry into the peritoneal cavity. The literature reported incidences of Veress needle (VN) injuries of e.g. $0.31 \%$ and $0.23 \%$. In a 2010 national survey of laparoscopic entry techniques in the Canadian General Surgical practice, 57.3\% of respondents had either experienced or witnessed a serious laparoscopic entry complication like bowel perforation and vascular injury. As those complications are potentially life threatening and should be avoided at all costs, improving safety of this initial action is paramount.

Methods Based on a bare minimum design approach with focus on function expansion of existing components, a new Safety mechanism was developed for the $\mathrm{VN}$ that decreases the risks of $\mathrm{VN}$ overshooting. The mechanism works by preventing the puncturing acceleration of the tip of the VN by decoupling the surgeon's hand from the VN immediately after entering the abdomen.

Results Based on a set of requirements, a first prototype of the $\mathrm{VN}+$ with force decoupling safety mechanism is presented and evaluated on an ex vivo porcine abdominal wall tissue model in a custom setup. The experiments conducted by two novices and one experienced surgeon indicated a significant difference between the attempts with a standard, conventional working $\mathrm{VN}$ (41.4 mm [37.5-45 mm]) and VN+with decoupling mechanism (20.8 mm [17.5-22.5 mm]) of $p<0.001$.

Conclusion A new decoupling safety mechanism was integrated successfully in a standard VN resulting in a $\mathrm{VN}+$. The results from the pilot study indicate that this new $\mathrm{VN}+$ reduces overshooting with a minimum of $50 \%$ in a standardised ex vivo setting on fresh porcine abdominal wall specimens.
\end{abstract}

Keywords Laparoscopy $\cdot$ Entry technique $\cdot$ Veress needle $\cdot$ Safety Mechanism

Laparoscopic surgery is a widely performed technique that has replaced open surgery as the gold standard in general surgery, gynaecology, and urology [1]. In general, three

Tim Horeman-Franse

t.horeman@tudelft.nl

1 Department of BioMechanical Engineering, Faculty of Biomedical Engineering, University of Technology Delft, Mekelweg 2, 2628 CD Delft, The Netherlands

2 Department of Surgery, University Medical Centers Amsterdam, Location VUMC, De Boelelaan 1117, 1081 HV Amsterdam, The Netherlands

3 Engineering Department, ProVinci Medtech, 2631 CM Nootdorp, The Netherlands

4 Department of General, Visceral, and Transplantation Surgery, Heidelberg University Hospital, Im Neuenheimer Feld 420, 69120 Heidelberg, Germany types of methods are used to gain initial access to the peritoneal cavity to establish pneumoperitoneum. The first, the so-called open technique as described by Hasson in 1971, requires a direct incision through the abdominal wall prior to the trocar insertion [2]. The second, the technique using a so-called optical trocar relies on the view through the tip of a bladeless trocar whereby the different layers of the abdominal wall can be seen during introduction of this trocar, thereby visually identifying entry into the abdomen [3]. The third technique, the closed technique, uses the Veress needle (VN) to gain abdominal distention prior to the first trocar insertion [4]. Contrary to the open and optical trocar technique, the closed technique with the $\mathrm{VN}$ involves a blind insertion directly into the peritoneal cavity $[1,5,6]$. Combinations of the three main initial access types also exist, e.g. the VN combined with the optical trocar technique. 
Complications that occur in laparoscopic surgery are often associated with the initial entry into the peritoneal cavity [5]. The literature reports incidences of $\mathrm{VN}$ injuries of e.g. $0.31 \%$ [1] and $0.23 \%$ [7]. An overview can be found in the Cochrane database [5]. In a 2010 national survey of laparoscopic entry techniques in the Canadian General Surgical practice, $57.3 \%$ of respondents had either experienced or witnessed a serious laparoscopic entry complication [8]. Complications like bowel perforation and vascular injury, can potentially be life threatening and should be avoided at all costs. Hence, improving safety of this initial action is paramount.

Up till now there has been no consensus on the safest method of the two techniques for initial entry into the peritoneal cavity [5]. In a study by Molloy et al. the VN was used in $81 \%$ of gynaecological laparoscopic operations and in $48 \%$ of general surgery procedures [6]. The use of the $\mathrm{VN}$ is probably popular because of its simplicity and effectiveness. It involves making a small incision in or near the umbilicus or in the left upper quadrant of the abdomen and then, in a blind fashion, putting the needle through the subcutaneous tissue, abdominal wall and the parietal peritoneum into the abdominal cavity. The VN technique is based on the ability of its blunt inner stylet to spring forward (spring loaded) and to cover the sharp bevelled tip of the outer cannula when resistance diminishes after all tissue layers are passed (Fig. 1). However, when the blunt tip approaches the tissue with too much speed and force, tissue damage still can occur. Hence, the surgeon cannot totally rely on this mechanism and therefore needs to develop a sense of the appropriate angle of insertion and the appropriate force to successfully puncture through the abdominal wall without overshooting into the underlying organs. The risk of damaging the underlying tissue with the tip of the cannula becomes high, when the reaction force that is generated by the abdominal wall drops to nearly zero (inside the abdominal cavity) in an instant. This immediate loss of resistance on the tip of the $\mathrm{VN}$ after puncturing causes acceleration of the needle towards the underlying tissues due to the slow reaction of the human control system $[9,10]$, absence of stiff lower arm/hand support and relatively large mass of the arm $[11,12]$. Therefore, skilled and safe use of the VN requires a long learning curve to achieve the best possible instrument handling to prevent overshoot. Moreover, every patient's abdomen presents unique operating conditions, of which the specifics are unknown to the surgeon prior to the operation. These include the presence of adhesions, positions of the underlying tissues and viscera and the thickness of the abdominal wall.

\section{State-of-the-art}

To solve the problem of puncturing underlying tissue during $\mathrm{VN}$ insertion, some straightforward modifications were suggested that limit overshooting by allowing the surgeon to set the maximal insertion depth [13]. Unfortunately, the tissue layer thickness is not always known and layers are

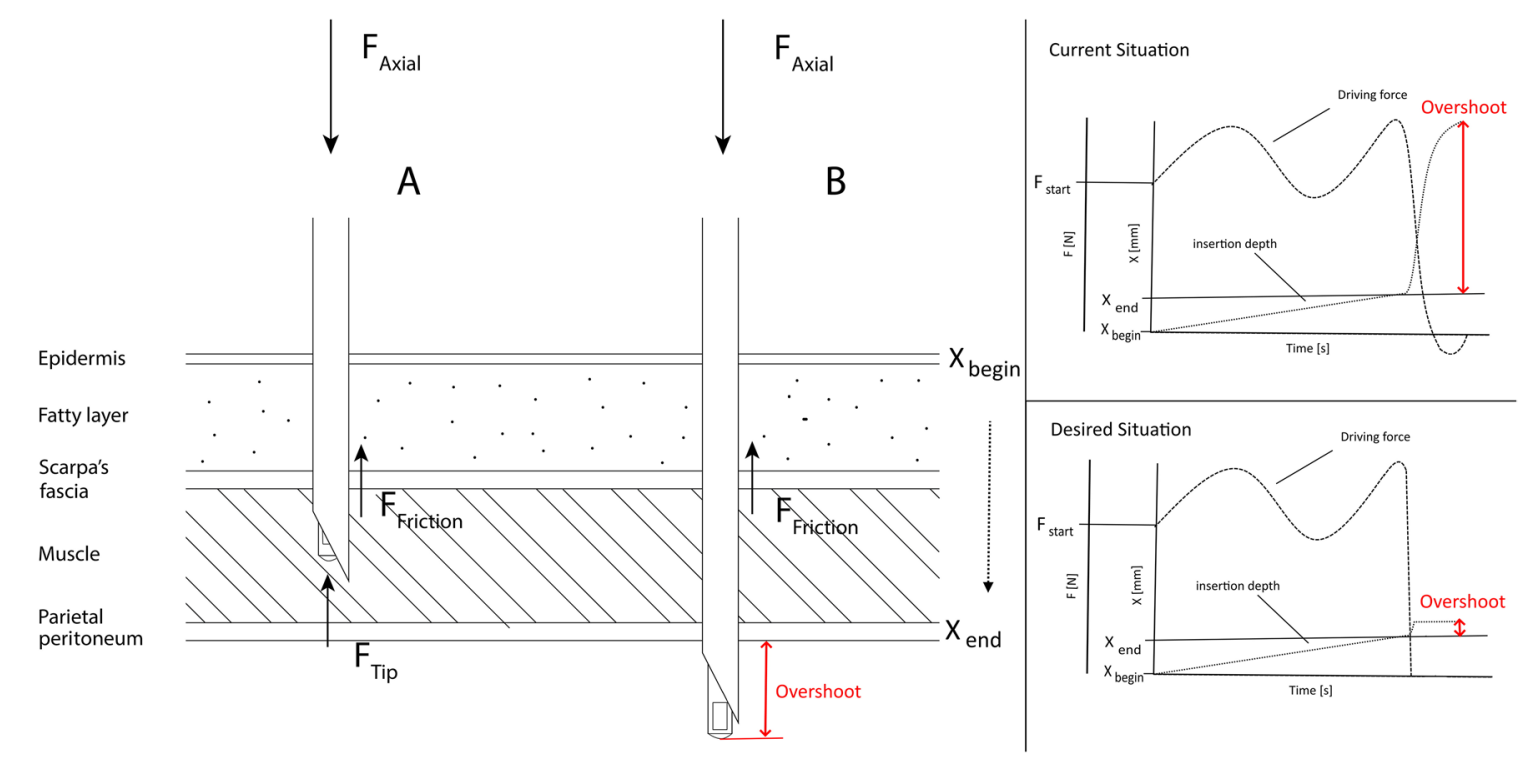

Fig. 1 Schematic figure of the Verres needle (VN) technique and the layers of the abdominal wall. Left: In A the sharp bevel tipped cannula and blunt stylet are penetrating the abdominal wall. In $\mathbf{B}$ the parietal peritoneum has been punctured and the tip and stylet are in the abdominal cavity. Right upper graph: Typical driving force moving the needle further into the cavity after penetrating the last layer.
Right lower graph: Desired driving force (dashed lines), reduced immediately to zero after penetrating the last layer not moving the needle further into the cavity, this limits the insertion depth (dotted lines). The amount of overshoot is indicated in red (Color figure online) 
flexible making adjustment of the insertion depth difficult. Another approach was to allow overshooting but to fixate the Veress mechanism as soon as it shoots in position [14]. However, even a blunt stylet with locked outer cannula can damage internal structures. Some research groups focused on expanding the blunt area of the tip directly after insertion to reduce the stress when the tip hits underlying organs or structures $[1,15]$. Other work demonstrates the capability to acquire information about intracorporeal tissue tool interactions of the VN tip, utilizing acoustic emissions or optic information recorded at the extracorporeal end of the needle $[16,17]$. Following a different approach, another group created a vacuum cup around the VN. When sucking the air out of the cup, the abdominal wall is lifted away from the critical structures when the VN penetrates the tissue [18]. Although good results are presented, the surgeon does lose manipulation flexibility as the configuration of the needle is fixed. Instead of changing the mechanism's functionality, some researchers suggest that providing additional information about pressure or vacuum on a visual scale helps reduce fatal organ damage [19-21]. Unfortunately, the effect of those indicators remains unknown. Finally, overshooting can be prevented by introducing a faster control system. This can be done by linking a robot arm with haptic sensation to the VN's body that generates the driving force [22]. Although some of those results show interesting results, the complex nature of these systems and the impact on workflow does jeopardize broader acceptance.

An alternative way to decrease the risks of overshooting of the $\mathrm{VN}$ is to reduce the acceleration of the tip after puncturing as quickly as possible. For this, a new mechanism needs to be developed that immediately removes the driving force (Fig. 1, right lower graph) as soon as the tip enters the cavity.

Based on a set of requirements, a first prototype of the $\mathrm{VN}+$ with force decoupling safety mechanism is presented and evaluated on an ex vivo abdominal wall tissue. The experiments were conducted by two novices and one experienced surgeon.

\section{Methods}

\section{Requirements}

After a thorough analysis of the problem, the following requirements were set for a new safer design of the $\mathrm{VN}$ :

1) Decrease acceleration after the abdominal wall has been punctured.

2) Keep the original VN functions (cannula with sharp tip, blunt inner stylet and spring loaded).
3) The haptic sensation and change in workflow during insertion should not add any risk to the procedure.

4) Device must be suitable to be sterilized in the Central Sterilization Department (CSD).

5) Compatible with multiple $\mathrm{VN}$ designs.

\section{Decrease acceleration}

A force and motion analysis has been performed to understand the mechanics behind the VN needle puncture. The needle insertion procedure contains the following phases that are taken from Van Gerwen [23]; (a) No interaction; (b) Boundary displacement; (c) Tip insertion; (d) Tip, cannula and stylet insertion. The important forces are the axial forces acting on the stylet/cannula tip, and the friction forces acting on the side of the cannula. When the $\mathrm{VN}$ is passing through the abdominal wall, the axial force can be described as: $F_{\text {axial }}=F_{\text {tip }}+F_{\text {friction }}$ (Fig. 1A). Once the last fascia and muscle layers have been punctured, the force on the stylet/ cannula tip $\left(F_{\text {tip }}\right)$ reduces instantly to zero. At this moment $F_{\text {friction }}$ is much lower than $F_{\text {axial }}$ and the needle accelerates towards the underlying tissues (Fig. 1B). Because the stiff fascia requires a high force to puncture [24] and the composition of the layers as well as a number of fascia layers differ per region [25], it becomes more difficult to predict the VN's behaviour and to prevent overshoot.

\section{Keep original VN functions}

The original spring action safety feature of the original VN were kept because of the simplicity of the design and surgeons already rely on this feature during use. After insertion, the $\mathrm{VN}$ is used to establish a pneumoperitoneum and therefore it should allow for $\mathrm{CO}_{2}$ insufflation to the peritoneum.

\section{The haptic sensation and change in workflow during insertion should not add any risk to the procedure}

The sequence in which the needle is activated, by applying pressure on the needle tip followed by movement of the inner stylet according to a known force displacement characteristic, is often programmed deep in the surgeon's haptic system. All changes that influence the workflow or haptic sensation during insertion through the different abdominal layers should be evaluated. If these properties are changed, the face and construct validity of the needle should be proven again.

\section{Suitable for sterilization in the CSD}

The device should be able to be cleaned and processed in the CSD within a reasonable timeframe [26]. 


\section{Compatible with multiple VN designs}

The mechanism should work with all VN needle diameters.

\section{Design approach}

Winter et al. found that, to gain broad acceptance of a technical innovation in the medical field, a dedicated design approach is often required [27]. This can be accomplished by developing intuitive and maintenance-friendly instrumentation that can be used by surgeon's worldwide working in both developed and poorly resourced hospitals with basic CSSDs. We have, therefore, used a "bare-minimum design" methodology, with a strong focus on component interaction analysis and adding functions to standard components [26-28], in combination with a stepwise development and evaluation plan that involves all key users coming into contact with the $\mathrm{VN}+$ to create the new safety mechanism.

\section{Pilot study test setup}

The goal of the experiment is to compare the modified $\mathrm{VN}+$ with the conventional VN when used by inexperienced users in a simulated clinical setting. The ability to puncture through the abdominal layers of a porcine wall without potentially damaging organs below the abdominal wall is determined by measuring how much of the needle penetrated the abdominal wall. For objective assessment a digital camera was used to record each measurement and to determine the lowest point of the needle cannula after the trial. A laser engraver was used to add markings on the metal of the $\mathrm{VN}$ cannula to determine the absolute insertion distance during use. Figure 2 shows the test setup used to fixate the specimen during the experiment. Therefore three intact abdominal walls of 200 by $150 \mathrm{~mm}$ were collected at the abattoir, for this no IRB approval was required. The specimens were directly frozen at -20 degrees for a maximum of three days prior to testing. Two hours before the experiments started a heating element was used to heat the samples to 37 degrees. The test setup consisted of a table with two independent Plexiglas plates of 300 by $200 \mathrm{~mm}$. An opening of $60 \mathrm{~mm}$ was cut in the bottom plate that allowed tissue to be pressed downwards whilst following the movement of the needle tip during insertion. Two additional rims with inner diameters of 80 and $100 \mathrm{~mm}$ were glued on the bottom plate around the hole for extra grip on the wall after fixation. The top plate had a raster of nine square openings each, measuring $10 \mathrm{~mm}$ square with a $2 \mathrm{~mm}$ distance between each opening. The raster prevented the participant from using the same hole twice during the experiment and kept the data reliable. The sample was installed by the observer after making the first incisions in the skin. After placement of the sample on the bottom plate, the top plate was placed and loosely tightened

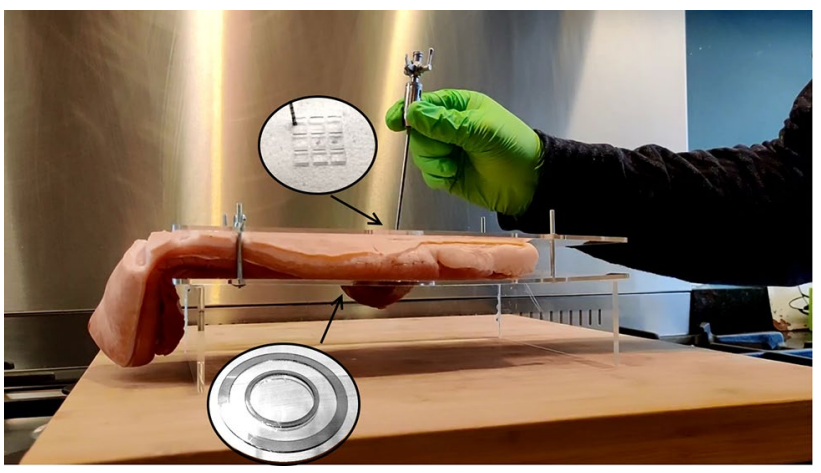

Fig. 2 Pilot study setup consisting of a support plate with a round hole and top plate with a raster of nine square holes. The nine holes prevent that a single location on the incision is used multiple times. The round hole allows tissue to deform and the needle tip to pass. Around the round opening rims were glued to increase the grip on the tissue. Four wire taps with butterfly nuts were used to tighten the tissue between the two plates

by 4 flinders nuts. A wooden block was placed under the tissue to stabilise the setup and to prevent contact between the tip and metal table.

\section{Measurement protocol}

To validate the setup and needle, experiments were executed by two novices and one experienced surgeon in VN placement. First, the participant got oral instructions. The experimenter explained to the participant that there are 2 modified versions of the $\mathrm{VN}$, to reduce demand characteristics and bias. The participant is shown how to correctly hold the VNs and is given the task to puncture through the tissue layers and to reduce the risk of overshooting as much as possible without touching the construction of the setup. The participant was asked to put the tip of the $\mathrm{VN}$ on the top of the top layer in the incision and to start the procedure. It is explained not to remove pressure from the needle until the needle passed all layers and reached the "cavity". Before the measurements started, all participants could ask questions and were allowed to investigate and test the VNs so that the principles were fully understood. Insertion of the needle was replicated 10 times with the spring-loaded safety mechanism activated $(\mathrm{VN}+)$ and 10 times without (VN). The participants were asked to switch the approach between each needle insertion. When all openings were used, a new tissue area was selected and the tissue relocated accordingly. Each participant used a new tissue area. In a second session, an experienced surgeon who had performed over $500 \mathrm{VN}$ procedures was asked to use the needle three times with the safety mechanism activated and three times without. The surgeon was asked to position and hold the tissue as realistic as possible before inserting the needle. 


\section{Data comparison and statistics}

Video footage was used by the observer to determine the insertion distance and a screenshot was made. From this data, the number of visual markings was counted and the true insertion depth was calculated in Excel based on the known distance between each marking. After the participants completed the tests, any differences in maximum insertion depth between the "conventional" $(\mathrm{VN})$ and "modified" $(\mathrm{VN}+)$ groups were determined with a Mann-Whitney test (SPSS v16, SPSS, Inc., Chicago, IL) as the data were not normally distributed. A $p$ value $<0.05$ was considered a significant difference.

\section{Results}

\section{Prototype VN+ design}

Figure 3 shows a drawing of the $\mathrm{VN}+$ with new safety decoupling mechanism and a conventional VN. The VN+ (Fig. 3Top) is based on this conventional VN (Fig. 3-Below) and has an additional lever mechanism, and grip component with flexor and some alignment pins.

Figure 4 indicates how the $\mathrm{VN}+$ mechanism functions during use. In the first phase, no external forces are present on any of the components and the spring-loaded lever finger is resting on top of the hook that connects the grip component to the base of the needle. In this phase it is not possible to slide the grip component axially away from the $\mathrm{VN}+$ 's body. When the $\mathrm{VN}+$ is inserted into tissue the inner stylet of the $\mathrm{VN}+$ slides upwards due to the axial force on the tip in the second phase. Now the spring-loaded lever finger

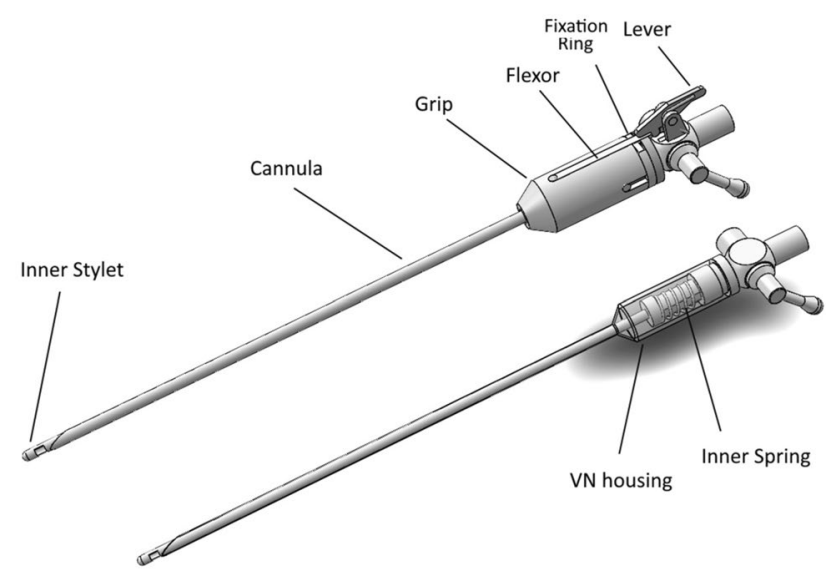

Fig. 3 Top, VN + design with additional Lever mechanism and Grip with Flexor that slides over the modified VN housing. At the end of the Flexor a hook is welded that locks the Grip behind the fixation ring of the modified VN housing. Below, a standard VN design with transparent $\mathrm{VN}$ housing showing the Inner spring that applies force on the Inner stylet when it moves relatively to the Cannula
(Phase 2, a) moves downwards relatively to the stylet in a new resting position. In the third phase, when the parietal peritoneum has been punctured the axial reaction force on the stylet instantly drops to zero. This causes the inner spring inside the $\mathrm{VN}+$ housing to deform back to its original state pushing the inner stylet back to its starting position. During this movement the finger of the spring loaded lever moves under the hook of the leaf spring (dark blue) disconnecting the grip from the $\mathrm{VN}+$. When the grip is disconnected from the rest of the needle in the fourth phase, the surgeon's hand and grip accelerate away from the $\mathrm{VN}+$ housing. Hence, the principle of the added safety mechanism is that the potential energy of the surgeon's arm, when applying a force on the $\mathrm{VN}+$, the grip is instantly decoupled from the rest of the $\mathrm{VN}+$ when the cannula tip penetrated the last tissue layer of the abdominal wall. Instead of driving the cannula further into the cavity, this potential energy is now safely removed from the needle as only the grip accelerate towards the abdomen whilst the rest of the $\mathrm{VN}+$ remains in position.

The working principle of the $\mathrm{VN}+$ decoupling mechanism is based on differences between the cannula tip force, tissue friction force and spring force acting on the stylet. To assure fast decoupling and a strong reliable system, the flexor (Fig. 4 and 5 dark blue) should not be too thick or thin. Therefore the maximum flexor thickness was determined with Formula 1. Supplemental file 1 shows that the maximum flexor thickness can be $0.4 \mathrm{~mm}$ when using the standard VN spring, the flexor length and width 20 and $2 \mathrm{~mm}$, respectively, the hook angle and height 30 degrees and $2 \mathrm{~mm}$, respectively, and stainless steel as flexor material.

$$
\begin{aligned}
& h=\sqrt[3]{\frac{12 \times(\mathrm{Fl}-\mathrm{Ff} t) \times L^{3}}{3 E u b}} \\
& \mathrm{Fl}=F a / \tan \theta \\
& F a=k \times x \\
& \mathrm{Fft}=\mu \times \mathrm{Ft}
\end{aligned}
$$

$h$ is the thickness flexor, $\mathrm{Fa}$ is axial force on flexor tip, $\mathrm{Fl}$ is lifting force hook, Ft is friction force tissue around needle, Fft is friction force between hook and ring, $\theta$ is hook angle flexor tip in degrees, $L$ is length of the flexor, $E$ is the elasticity modulus Stainless steel, $u$ is elevation hook for disconnection, $b$ is width flexor, $k$ is spring rate $\mathrm{VN}$ housing spring, $x$ is spring indentation and $\mu$ is the friction coefficient.

Figure 5 shows a cross sectional area of the functional components of the $\mathrm{VN}+$. All relevant forces, dimensions and parameters of Formula 1 are indicated in the figure.

The VN+ prototype in Fig. 6 was manufactured out of a standard conventional working $\mathrm{VN}$ needle for laparoscopic surgery made from stainless steel (SS316, TROKAMED GmbH, Kleine Breite 17, 78187 Geisingen, Germany). Modifications were done at Van Straten Medical (Rijnzathe 
Fig. 4 Basic functioning of the $\mathrm{VN}+$ design. Phase 1 , the rest situation where the system is ready for use. Phase 2, during insertion the sharp cannula is exposed and the decoupling mechanism is armed automatically. Phase 3 , as soon as the cannula tip penetrates the last tissue layer the stylet shoots out and decouples the grip component when the lever finger lifts the hook (dark blue) over the fixation ring (magenta). Phase 4 , the force exerted by the hand on the needle is instantly decoupled resulting in acceleration of the grip component opposite to the housing (Color figure online)
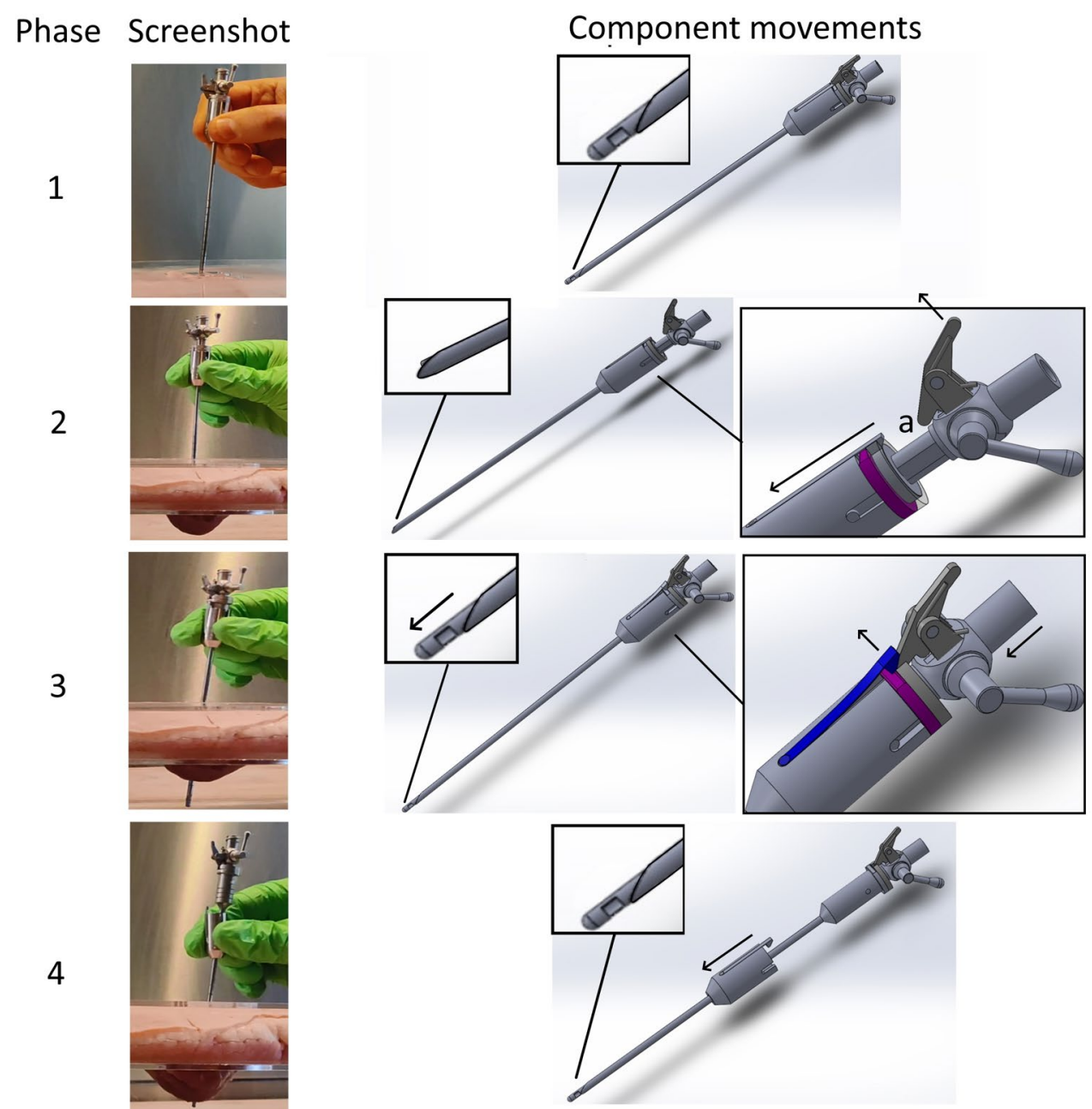

shows videos with the $\mathrm{VN}+$ with decoupling mechanism. The total experiment including the introduction, instructions and tasks took a maximum of 30 min per participant. Figure 7 shows typical end-situations after inserting the standard VN and VN+ with decoupling mechanism. Supplemental File 3 shows the total insertion depth data as presented in Fig. 8.

The Mann-Whitney test indicated a significant difference between the attempts with a standard, conventional working VN (41.4 mm [37.5-45 mm]) and VN+ with decoupling mechanism $(20.8 \mathrm{~mm}$ [17.5-22.5 mm]) of $p<0.001$. The participants did observe differences in force needed to penetrate the different layers and the number of force build ups during the insertion task. When using the conventional VN the wooden block under the setup was not touched by the needle tip. In the rest of the attempts with the standard VN the block was firmly hit and a sound was heard by the observers. When using the $\mathrm{VN}+$ the wooden block was not reached by the needle tip. From the 26 attempts, only one attempt failed when the standard $\mathrm{VN}$ jammed and the inner cannula did not move back after 


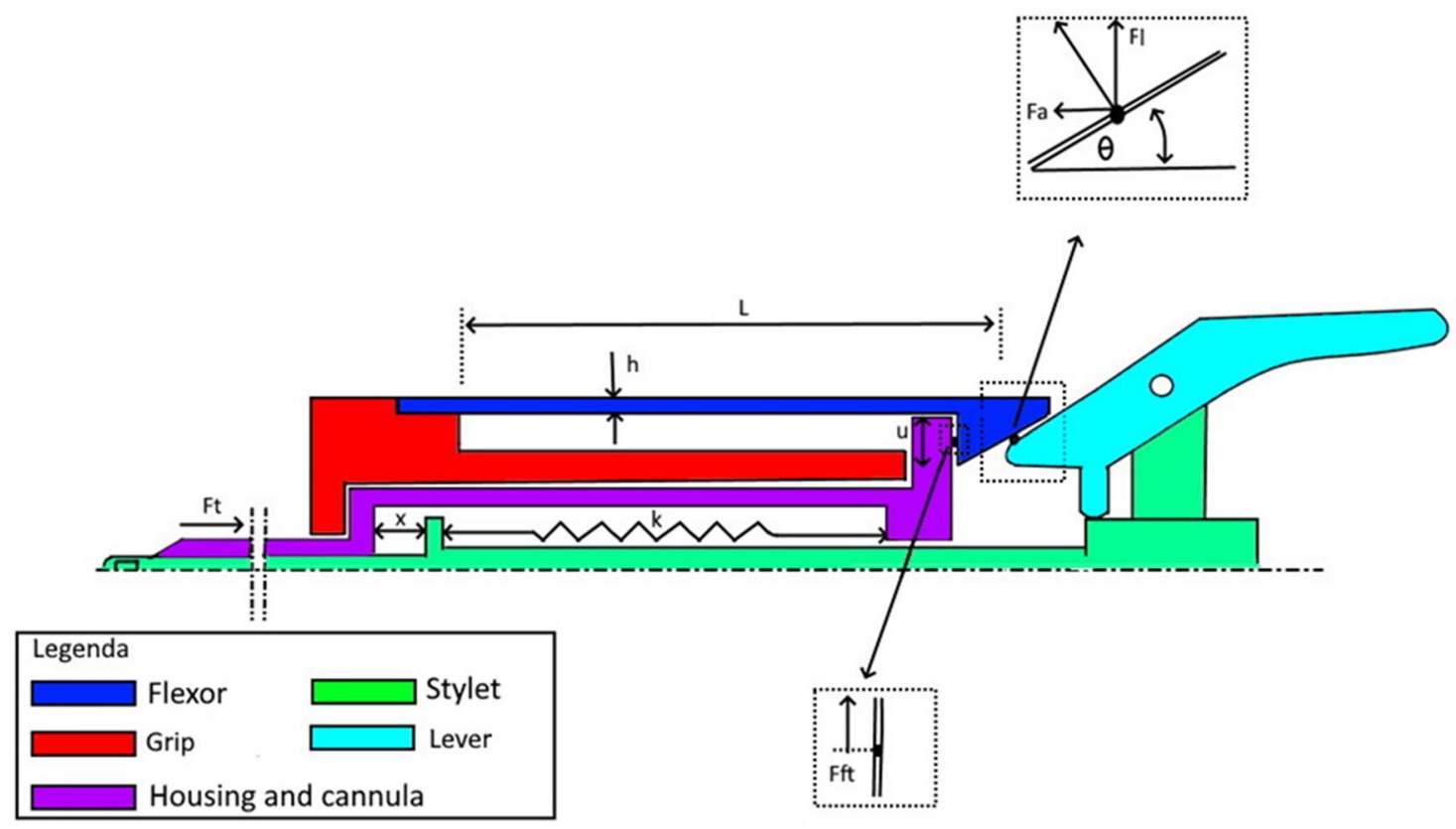

Fig. 5 Schematic drawing of the $\mathrm{VN}+$ safety system. The flexor of the grip (dark blue) is lifted upwards by the lever (light blue) when the inner stylet (green) shoots to the left when all tissue layers are passed. When the flexor is lifted, the grip component (red) is decoupled from the $\mathrm{VN}+$ and accelerating away from the housing (Color figure online)
Fig. 6 First prototype of the $\mathrm{VN}+$ with decoupling mechanism. Additionally, extra protection rims (a) and a cap (b) were placed on the grip component to prevent that the user interferes with the flexor element or guidance pin

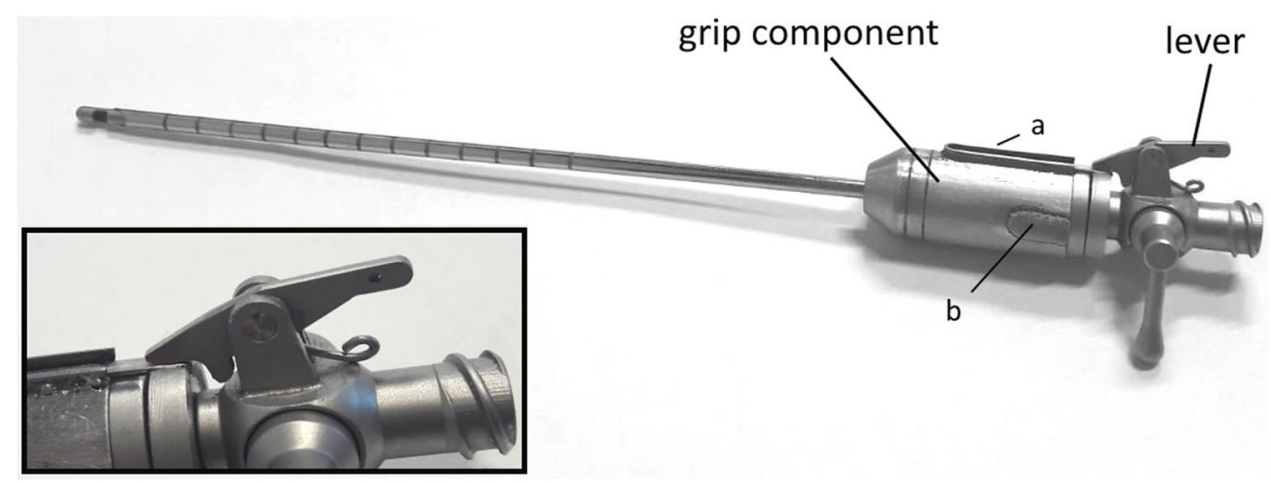

penetrating the last layer due to tissue residue build-up between stylet and cannula.

\section{Discussion}

A new decoupling safety mechanism was integrated successfully in a standard $\mathrm{VN}$ resulting in a $\mathrm{VN}+$ that complies with all current cleaning requirements [28]. The mathematical models successfully related the forces acting on the flexor and hook to the flexor dimensions, indicating that the internal spring stiffness inside the housing of the $\mathrm{VN}$ and angle of the hook are important factors of influence. From a functional point of view the flexor should be as thin as possible. However, from a practical point of view the system
Fig. 7 Typical end-situations after inserting the conventional VN (left) and $\mathrm{VN}+$ with decoupling mechanism (right) 
Fig. 8 Pilot study data. Left, maximal insertion depth when using the standard VN. Right, maximal insertion depth when using the $\mathrm{VN}+$ with decoupling mechanism

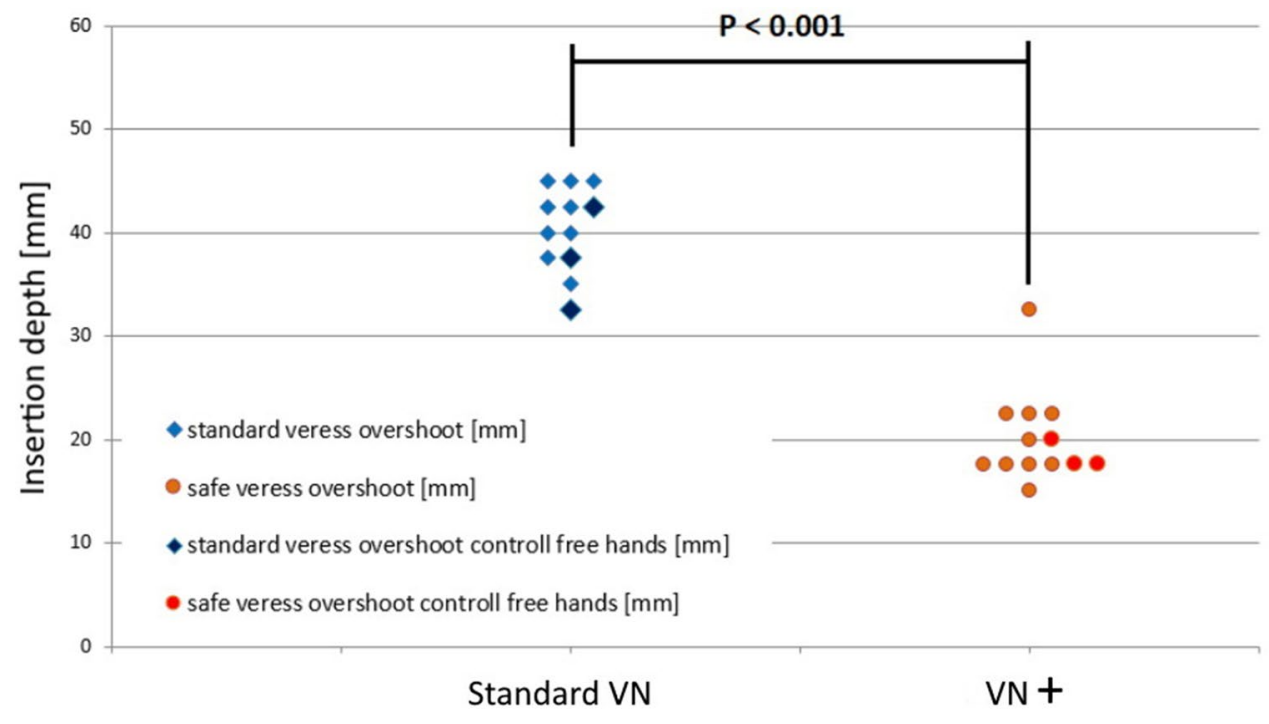

As soon as the participants started to trust the mechanism and were not afraid to apply force during insertion, the system did not result in early detachment anymore.

The results from the 6 additional trials conducted by the experienced surgeon on a loose abdominal wall sample did not differ from the earlier measurements. This indicates that the fixation of the abdominal wall did not influence the measurements. As the $\mathrm{VN}+$ showed real potential during the pilot study, our team plans to fully characterise the system during a human cadaver study with representative tissue properties to ensure that all risks are accounted for.

Although the safety mechanism was integrated in a $\mathrm{VN}$, other needle and trocars systems can benefit from this approach as well. In a war zone, lifesaving access systems, used to access major blood vessels to prevent or treat haemorrhagic shock or to establish access to the airway/trachea, are often used by less experienced medical personnel working under stressful situations. Further studies should indicate if integration of a similar safety mechanism in those lifesaving needle systems can reduce failure.

\section{Limitations}

Since this was a preclinical evaluation several potential differences to the real clinical situation can be discussed. Some surgeons adopt different techniques to avoid overshooting when using the $\mathrm{VN}$, e.g. placing the wrist of the hand on the patient's skin and manipulating the VN only with the force of the fingers and wrist instead of the whole arm to reduce overshooting; slowing down between each layer of the abdominal wall to have higher control and to reduce overshooting. These strategies, in combination with conventional $\mathrm{VN}$ and $\mathrm{VN}+$, should be addressed in further usability studies. In addition, the current evaluations were performed on an ex vivo porcine composite abdominal wall 
model with standardized settings. Although the surgeon suggested that the porcine tissue properties felt realistic, the results will have to be transferred and validated in human cadaveric settings as well as live animal models before use of $\mathrm{VN}+$ on patients. The use of the $\mathrm{VN}+$ will also have to be assessed for different entry points to the abdomen such as "Palmers" point in the left subcostal region as well as midline and periumbilical regions and lower abdomen to account for different tissue resistance settings with regards to distance to bony- and other structures [7]. Many surgeons employ strategies like pulling up the skin with their hands or forceps to lift the abdominal wall away from abdominal organs to reduce risk of damage [29]. Additional comparison studies should indicate if this behaviour resembles the preclinical setting of the present study with a fixed size exit hole of several centimetres or that modification of the setup is needed to increase realism. Still, the current evaluations show enormous potential of $\mathrm{VN}+$ for improving safe access to the abdomen for laparoscopic surgery by reducing overshooting and subsequent risk of organ damage. Therefore further development and investigation is warranted.

\section{Conclusion}

The bare minimum design approach with focus on function expansion of existing components resulted in a new safety mechanism that was successfully integrated in an existing VN. The results from the pilot study indicate that this new VN+reduces overshooting with a minimum of $50 \%$ in a standardised ex vivo setting on fresh porcine abdominal wall specimens. Optimisation could reduce the mass of the $\mathrm{VN}+$ components and friction between components which could further reduce the insertion depth. Although the results are very promising, additional experiments on all known insertion locations and on other cadaver material are needed to investigate how the obtained results translate towards a more clinically real setting.

Supplementary Information The online version contains supplementary material available at https://doi.org/10.1007/s00464-021-08603-x.

Acknowledgements The authors like to thank Kars Moens from the Leidse Instrumentmakers School for manufacturing of the stainless steel components and assembly of the Safe VN. We thank the company Van Straten Medical for providing us with new VN needles for modification and testing.

\section{Declarations}

Disclosures David Cefai has a patent pending on the $\mathrm{VN}+$, Roelf Postema, Bart van Straten, Rein Miedema, Latifa Lesmana Hardjo, Jenny Dankelman, Felix Nickel and Tim Horeman-Franse have no conflicts of interest or financial ties to disclose.
Open Access This article is licensed under a Creative Commons Attribution 4.0 International License, which permits use, sharing, adaptation, distribution and reproduction in any medium or format, as long as you give appropriate credit to the original author(s) and the source, provide a link to the Creative Commons licence, and indicate if changes were made. The images or other third party material in this article are included in the article's Creative Commons licence, unless indicated otherwise in a credit line to the material. If material is not included in the article's Creative Commons licence and your intended use is not permitted by statutory regulation or exceeds the permitted use, you will need to obtain permission directly from the copyright holder. To view a copy of this licence, visit http://creativecommons.org/licenses/by/4.0/.

\section{References}

1. Nevler A, Har-Zahav G, Rosin D, Gutman M (2016) Safer trocar insertion for closed laparoscopic access: ex vivo assessment of an improved Veress needle. Surg Endosc 30:779-782

2. Hasson HM (1971) A modified instrument and method for laparoscopy. Am J Obstet Gynecol 110(6):886-887

3. Tanaka C, Fujiwara M, Kanda M, Murotani K, Iwata N, Hayashi M, Kobayashi D, Yamada S, Kodera Y (2019) Optical trocar access for initial trocar placement in laparoscopic gastrointestinal surgery: a propensity score-matching analysis. Asian J Endosc Surg 12(1):37-42. https://doi.org/10.1111/ases.12484

4. Veress J (1938) Neues Instrument zur Ausführung von Brustoder Bauchpunktionen und Pneumothoraxbehandlung. Deutsch Med Wochenschr 40:1480-1481

5. Ahmad G, Duffy JMN, Phillips K, Watson A (2019) Laparoscopic entry techniques. Cochrane Database of Syst Rev. https:// doi.org/10.1002/14651858.CD006583.pub5

6. Molloy D, Kaloo PD, Cooper M, Nguyen TV (2002) Laparoscopic entry: a literature review and analysis of techniques and complications of primary port entry. Aust N Z J Obstet Gynaecol 42:246-253

7. Azevedo JLMC, Azevedo OC, Miyahira SA, Miguel GPS, Becker Jr OM, Hypolito OHM et al (2009) Injuries caused by Veress needle insertion for creation of pneumoperitoneum: a systematic literature review. Surg Endosc 23:1428-1432

8. Compeau C, McLeod NT, Ternamian A (2011) Laparoscopic entry: a review of Canadian general surgical practice. Can J Surg 54:315-320

9. Haggard P, Wing AM (1991) Remote responses to perturbation in human prehension. Neurosci Lett 122(1):103-108

10. Hasan Z (2005) The human motor control system's response to mechanical perturbation: should it, can it and does it ensure stability? J Mot Behav 37(6):484-493

11. Dolan JM, Friedman MB, Nagurka ML (1993) Dynamic and loaded impedance components in the maintenance of human arm posture. IEEE Trans Syst Man Cybern Syst 23(3):698-709

12. Rahman MM, Ikeura R, Mizutani K (2002) Investigation of the impedance characteristic of human arm for development of robots to cooperate with humans. JSME Int J Ser C 45(2):510-518

13. Whitley KW, Adhikary SD (2015) U.S. Patent Application No. $14 / 660,302$

14. Wortrich TS (1994) U.S. Patent No. 5,364,365. Washington, DC: U.S. Patent and Trademark Office

15. DuBois K, Ryan P, Joanis M (2019) The Theia Soteria: alternative design for safer initial entry during laparoscopic procedures. Honors Theses. 2289. https://digitalworks.union.edu/ theses/2289. Accessed Mar 2020

16. Schaufler A, Sühn T, Esmaeili N, Boese A, Wex C, Croner R, Illanes A (2019) Forcematic differentiation between Veress needle 
events in laparoscopic access using proximally attached audio signal characterization. Curr Dir Biomed Eng 5(1):369-371

17. Schaller G, Kuenkel M, Manegold BC (1995) The optical "Veress-needle"-initial puncture with a minioptic. Minim Invasive Ther Allied Technol 3(1):55-57

18. Greenberg JA (2008) LapCap ${ }^{\mathrm{TM}}$. Rev Obstet Gynecol 1(2):84

19. Gresl C, Kloeckl TL (1992) U.S. Patent No. 5,104,381. Washington, DC: U.S. Patent and Trademark Office

20. Guillermo Z (1925) U.S. Patent No. 1,527,291. Washington, DC: U.S. Patent and Trademark Office

21. Tovey HJ, Autry SD (1995) U.S. Patent No. 5,470,316. Washington, DC: U.S. Patent and Trademark Office

22. Nillahoot N, Suthakorn J (2013) Development of Veress needle insertion robotic system and its experimental study for force acquisition in soft tissue. 2013 IEEE international conference on robotics and biomimetics (ROBIO). IEEE, Piscataway, pp 645-650

23. Van Gerwen DJ, Dankelman J, van den Dobbelsteen JJ (2012) Needle-tissue interaction forces-a survey of experimental data. Med Eng Phys 34(6):665-680

24. Jansen FW, Kapiteyn K, Trimbos-Kemper T, Hermans J, Trimbos JB (1997) Complications of laparoscopy: a prospective multicentre observational study. BJOG 104(5):595-600
25. Bogduk N, Macintosh JE (1984) The applied anatomy of the thoracolumbar fascia. Spine 9(2):164-170

26. Hardon SF, Schilder F, Bonjer HJ, Dankelman J, Horeman T (2019) A new modular mechanism that allows full detachability and cleaning of steerable laparoscopic instruments. Surg Endosc 33(10):3484-3493

27. Winter A, Govindarajan V (2015) Engineering reverse innovations: principles for creating successful products for emerging markets. Harv Bus Rev 93:80-89

28. Horeman T, Schilder F, Aguirre M, Kerkhoffs GMMJ, Tuijthof GJM (2015) Design and preliminary evaluation of a stiff steerable cutter for arthroscopic procedures. J Med Dev 9(4):044503

29. Garry R (1999) Towards evidence-based laparoscopic entry techniques: clinical problems and dilemmas. Gynaecol Endosc 8(6):315-326

Publisher's Note Springer Nature remains neutral with regard to jurisdictional claims in published maps and institutional affiliations. 